\title{
THE ROLE OF MAGNETIC FIELDS IN STAR FORMING REGIONS
}

\author{
L MESTEL \\ Astronomy Centre \\ University of Sussex \\ Brighton BN1 9QH, UK
}

\begin{abstract}
.
The flux from the galactic magnetic field alters radically the appropriate description of the equilibrium, collapse and fragmentation of the self-gravitating gas clouds that are the locale of star formation.
\end{abstract}

\section{Magnetism and Gravitation: Sub-critical and Super-critical Clouds}

When the idea of a dynamically important, large-scale galactic magnetic field was first put forward, some of the sceptics claimed that it would prevent the formation of stars. The argument went essentially as follows. Let the idealized local galactic background have uniform density $\rho_{0}=\mathrm{n}_{0} \mathrm{~m}_{\mathrm{H}}$ and be permeated by a uniform field ${\underset{\sim}{0}}_{0}$. A sphere of radius $R_{0}$ and so of mass $M=4 \pi \rho_{0} R_{0} 3 / 3$ after contracting to $a$ new radius $R$ exerts a mean gravitational force density $\simeq$ $\left(G M / R^{2}\right)\left(\rho_{0} R_{0}{ }^{3} / R^{3}\right)$. The same contraction increases the strength of the frozen-in field to $B=B_{0} R_{0}{ }^{2} / R^{2}$; and once $R<<R_{0}$, the radius of curvature $R$ of the locally distorted field lines implies a current density

$j=c\left|\nabla \times B_{\sim}\right| / 4 \pi \simeq c B / 4 \pi R$, and so a Lorentz force density $|j \times B / c| \simeq B^{2 / 4 \pi R}=$ $B_{0}{ }^{2} R_{0} 4 / 4 \pi \tilde{R^{5}}$. The ratio of forces $4 \pi G M \rho_{0} / B_{0}{ }^{2} R_{0}$ is thus independent of $R$. Since $R_{0}=\left(3 \mathrm{M} / 4 \pi \rho_{0}\right)^{1 / 3}$, a mass capable of indefinite gravitational contraction against the opposing magnetic forces must therefore exceed the "magnetic Jeans mass":

$M>\left(B_{0} / \rho_{0}{ }^{2 / 3}\right)^{3}\left(1 / G^{3 / 2}\right)\left(3 \frac{1}{2} / 16 \pi^{2}\right)=\left[\frac{B_{0} / 3 \times 10^{-6}}{\left(n_{0} / 1\right)^{2 / 3}}\right]^{3} 3 \times 10^{6} M_{0}$

(normalising in terms of currently accepted values).

The argument is in fact over-stated, and with its assumption of isotropic accumulation of gas somewhat misleading, since one may reasonably expect the field will tend to channel flow parallel to itself. It is more enlightening to begin by accepting that a roughly spheroidal cloud oblate about the direction of $\mathrm{B}_{0}$ has formed, with semi-major and minor axes $R, z$. A frozen-in field can be written $B$ $=B_{0} R_{0}{ }^{2} / R^{2}=F / \pi R^{2}$, where $R_{0}$ is the value of $R$ at which the field is undistorted, and $F$ is the flux trapped within the cloud. Once $R<<R_{0}$, the magnetic curvature yields a force density $\simeq B^{2 / 4 \pi z}=F^{2 / 4} \pi^{3} R^{4} z$, opposing $a$ gravitational force density $\simeq G M \rho / R^{2} \simeq G^{2} /(4 \pi / 3) R^{4} z$. The ratio of magnetic to 
gravitational force then yields the non-dimensional flux/mass parameter

$f=F / \pi^{2} G^{\frac{1}{2}} M$.

For indefinite gravitational collapse to be possible, $f$ must be less than a critical value $f_{c}$ of order unity, the precise value being only weakly dependent on the degree of flattening and the details of the flux distribution. Corresponding to $f_{c}$ there is a minimum mass $M_{c}=F / \pi^{2} G^{\frac{1}{2}} f_{c}$ for a given flux $F$. Equivalently, there is a minimum value for the ratio $G \frac{1}{2} \sigma / B$, where $\sigma=M / \pi R^{2}$ is the projected area density. If we again postulate that the cloud has formed under strict flux-freezing from the galactic background $\left(\rho_{0},{\underset{\sim}{\mathrm{B}}}_{0}\right)$, then the ratio $\mathrm{M} / \mathrm{F}$ defines an accumulation length $\mathrm{L}_{0}$ along $\mathrm{B}_{0}$ :

$\frac{M}{\mathbf{F}}=\frac{\sigma}{B}=\frac{\rho_{0} L_{0}}{B_{0}}$,

and the collapse condition becomes

$L_{0}>\left(L_{0}\right)_{c}=\left\{\frac{B_{0}}{G^{\frac{1}{2}} \rho_{0}}\right\} \frac{1}{\pi^{2} f_{c}} \simeq 700 \frac{\left(B_{0} / 3 \times 10^{-6}\right)}{\left(n_{0} / 1\right)}$ parsecs.

The contrast with the argument that assumes isotropic formation is now clear: no length-scale across the field enters the criterion. A thinner flux-tube contains a correspondingly smaller mass; but once the mass in a cylinder of length $>\left(L_{0}\right)_{c}$ has accumulated down the field into a spherical or oblate body, its self-gravitation will always exceed the maximum force exerted by the locally distorted magnetic field.

The value (4) is of same order as the minimum Rayleigh-Taylor-Parker instability wavelength (Parker 1969, Mouschovias 1974), so it may be argued that an accumulation length of the order of a kiloparsec is not necessarily an embarrassment. Nevertheless, one is led to wonder whether during violent cloud formation (eg following a huge input of energy into the interstellar medium from a supernova), strict flux freezing does hold, or whether ( $c f$. Sweet 1950, and many papers since, especially on dynamo theory) an effective turbulent resistivity yields net diffusion of gas across the field, so that $\left(\mathrm{L}_{0}\right)_{c}$ becomes a notional length. This suggests that one should keep separate the problem of cloud formation from that of the equilibrium, collapse and fragmentation of star-forming clouds.

The discussion has a natural bifurcation at this point.

Case A. Super-critical clouds, with $f<f_{c}$ and $M>M_{c}(F)$. Magnetic forces alone are unable to prevent collapse of the whole cloud. Further, as no length across $\underset{\sim}{\mathrm{B}}$ enters the collapse condition, a cylinder within a super-critical cloud contains enough mass to form a super-critical spherical or oblate fragment, following flow of the mass down the field lines (Mestel 1965). Thus fragmentation of a super-critical cloud is possible even under strict flux freezing, but the bodies so forming within a cloud that is just super-critical ( $f \quad f_{c}$ ) will themselves be "strongly magnetic". A markedly super-critical cloud would probably be difficult to observe, as it will collapse and perhaps fragment in about a free-fall time.

Case B. Sub-critical clouds, with $\mathrm{f}>\mathrm{f}_{\mathrm{c}}$. Even if the cloud is cool, weakly turbulent and slowly rotating, indefinite collapse in the two trans-field dimensions is prevented by the frozen-in flux; after dissipation of macroscopic kinetic energy, the cloud must settle into a state of approximate magneto-gravitational equilibrium in 
two dimensions, with the scale along the field determined by the thermal and turbulent energies (Mestel 1965; Strittmatter 1966; Mestel \& Paris 1984 for approximate treatments; Parker 1973, 1974; Mouschovias 1976a, b; Mestel \& Ray 1985; Tomisaka et al. 1988; Barker \& Mestel 1989 for detailed solutions). When $M \quad M_{c}(F)$, the trans-field radius is

$R \simeq R_{0}\left(1-\left(M / M_{C}\right)^{2}\right), \quad R_{0}=\left(F / \pi B_{0}\right)^{\frac{1}{2}}$

and the mean field strength

$B \simeq B_{0}\left(R_{0} / R\right)^{2} \simeq G^{\frac{1}{2}} M / R^{2}$.

When $M<<M_{C}$, then $R \simeq R_{0}$ and $B \simeq B_{0}-$ a slight distortion of the field suffices to generate the forces able to balance gravity. In either case, $\rho$ can increase by flow down the field if the cloud is cool and weakly turbulent. The B- $\rho$ relation observed will depend not only on the thermal and dynamical state of the gas, but also on the mass of the body considered.

The prediction of bodies held essentially in magneto-gravitational equilibrium (implicit in the Chandrasekhar-Fermi (1953) virial theorem) received its first observational support some twenty years ago. I recall writing to Dr Verschuur in 1968 after hearing that he had successfully picked up the Zeeman effect on the $21 \mathrm{~cm}$ line, asking him for both the densities and the estimated masses of his clouds. It was gratifying to learn that the kinematic, flux-freezing condition - high $B$ correlated with high $\mathbf{n}$ - and the dynamical condition - $M$ close to the virial estimate (6) - were both apparently fulfilled. For a resumé of the now overwhelming observational evidence, see Myers \& Goodman (1988 and this volume) and Troland (this volume).

A crucial feature that was not predicted is the typical Doppler width of molecular cloud lines, which is a clear indication of strong supersonic "turbulent" motions. The mean inferred speed $v_{t}$ must be substituted for the sound speed when estimating the typical scale $z$ along the field. If the cloud is markedly flattened, the Spitzer-Ledoux one-dimensional virial theorem is appropriate:

$v_{t}{ }^{2} \simeq 2 \pi G \rho z^{2} \simeq 3 \mathrm{GMz} / 2 \mathrm{R}^{2}$.

In a near-critical cloud ( $M \quad M_{C}$ ), equations (6-8) yield (with numerical factors of order unity ignored)

$B \simeq G^{\frac{1}{2}} \rho z \simeq \rho^{\frac{1}{2}} v_{t}$

independent of the cloud mass (Myers \& Goodman 1988). If the turbulence is strong enough to keep the cloud nearly spherical, the only changes are in the numerical factors. Thus in a cloud with $f$ close to $f_{c}$, the equilibrium achieved by the lateral adjustment of $B$ and $\rho$ and the longitudinal adjustment of $\rho$ inevitably yields by $(8)$ an Alfvén speed $v_{A}=B /(4 \pi \rho)^{\frac{1}{2}}$ close to the turbulent speed $v_{t}$. Equivalently (7) and (8) yield

$B \simeq v_{t}{ }^{2} / G^{\frac{1}{2}} z \simeq G^{\frac{1}{2}} \rho^{2 / 3} M^{1 / 3}\left[v_{t}{ }^{2} /(G M / R)\right]^{2 / 3}$.

Thus if $v_{t}$ is constant, comparison of near-critical clouds of different mass should yield $B \propto \rho^{\frac{1}{2}}$, whereas if $v_{t}$ is a constant fraction of the free-fall speed, from (9) $B$ 
$\propto \rho^{2 / 3} M^{1 / 3}$.

The most plausible suggestion for the turbulent motions is that they are essentially a field of torsional Alfvén waves propagating outwards along $\underset{\sim}{\mathbf{B}}$ with decaying amplitude (Arons \& Max 1975; Zweibel \& Josafatsson 1983; and others). The simplest illustrative example (Dewar 1970; Shu et al. 1987) has a mean field $\mathrm{B}_{0} \mathrm{z}$ and transverse waves with amplitudes $\mathrm{b}, \delta \mathrm{v}$ :

$\delta \underset{\sim}{B}=\underset{\sim}{b} \sin (k z-\omega t) \exp \left(-k_{1} z\right), \quad b \simeq\left(\delta v / v_{A}\right) B_{0}$.

The time average of the fluctuating Lorentz force density $\underset{\sim}{\mathbf{f}}=\mathbf{I} b$ $\left.\exp \left(-k_{1} z\right)\right|^{2}\left(k_{1} / 8 \pi\right)$. A non-zero damping constant $k_{1}$ is essential for a net input of momentum into the cloud, but the associated energy input need not be as heat but may be as macroscopic kinetic energy (eg rotational energy). Although there is evidence for flattened structures in some clouds (eg Sargent et al 1988), it appears that usually the force $f$ is able to keep the cloud moderately oblate, so it must be comparable with the lateral force density $B_{0}{ }^{2 / 4 \pi R}$, requiring $b \sim B_{0}$ with $\delta v \sim v_{A}$, and $1 / k_{1} \simeq R$. The source of the motions could be waves from sub-condensations that are being magnetically braked ( $c f$. below), or winds from proto-stars, or local expanding HII regions from hot stars newly arrived on the main sequence. It is also possible that cold disc-like clouds in magneto-gravitational equilibrium may be spontaneously unstable against the conversion of gravitational into kinetic energy. The essential point is that super-Alfvénic motions generated will dissipate rapidly through compressive shock formation, but in the linear approximation, density variations associated with the Alfvern waves are zero, so that even though the motions with $\delta \mathbf{v} \sim \mathbf{v}_{\mathbf{A}}$ will be highly supersonic there may not be rapid dissipation. An obvious difficulty is that the above analysis is directly applicable as a WKBJ approximation only if the semi-wavelength $\pi \mathrm{k}^{-1}$ is small compared with the scale of the cloud, but it is for small wavelengths that damping by ion-neutral collisions and second order steepening is most effective. A further constraint is that heat input from wave dissipation must not raise the cloud temperature above the observed maximum of $\simeq 20 \mathrm{~K}$ (Zweibel \& Josafatsson 1983). However, although more analysis is desirable, the theoretical picture of combined magnetic and Alfvénic turbulent support of molecular clouds remains eminently plausible.

\section{Flux leakage}

All observed stars are magnetically "weak". Any plausible inward extrapolation of the surface field of even the strongest observed magnetic star always yields internal field strengths such that

magnet ic energy/gravitational energy $\simeq\left(\mathrm{F} / \pi \mathrm{G}^{\frac{1}{2}} \mathrm{M}\right)^{2} \ll 1$.

It is quite impossible to account for this by again appealing to anisotropic mass accumulation under the flux-freezing constraint: to make $M / F$ sufficiently large, the accumulation length given by (3) would have to be ridiculously long (at least $40 \mathrm{kpc}$ ). There can be no doubt that gas in the observed strongly magnetic clouds must at some epoch lose the bulk of the flux threading it before it condenses into an observed main sequence or pre-main sequence star; but this catastrophic reduction of the primeval flux need not necessarily occur during the diffuse "star formation" epoch. Equally, a modest relaxation of strict flux freezing can affect profoundly the evolution of a cloud - eg by assisting fragmentation - while leaving the magnetic 
forces still dynamically important.

In a lightly ionized HI or molecular cloud, flux leakage occurs through the process called "plasma-drift" or (more often) "ambipolar diffusion". The Lorentz force exerted by the gravitationally distorted magnetic field acts directly on the charged component (the "plasma"), forcing it to drift relative to the neutral bulk. The drift velocity $\mathrm{v}_{\mathrm{d}}$ of the plasma plus the inductively-coupled $\underset{\sim}{\mathrm{B}}$-field is fixed by the balance between $\mathrm{j} \times \underset{\sim}{\mathrm{B}} / \mathrm{c}$ and the plasma-neutral friction (Mestel \& Spitzer 1956, and many papers sincẽ):

$\mathrm{v}_{\mathrm{d}}=(\underset{\sim}{\mathbf{j}} \times \underset{\sim}{\mathrm{B}} / \mathrm{c}) / \alpha\left(\mathrm{n}_{\mathbf{i}} / \mathrm{n}\right) \rho^{2}$

with $n_{i}$ and $n$ the respective numbers of ions and neutral particles. The frictional coefficient $\alpha=\left\langle\sigma_{\text {in }}\left(v_{T}\right)_{n}\right\rangle / m_{n}$, where $m_{n}$ is a mean neutral particle mass, $\sigma_{\text {in }}$ the momentum transfer collision cross-section, and $\left\langle\sigma_{\text {in }}\left(v_{\mathrm{T}}\right)_{n}\right\rangle$ the collision rate averaged over the thermal velocity distribution of the neutral particles; whence the number of collisions per second felt by an atomic or molecular ion (more massive than the neutral hydrogen and helium atoms or molecules) is $1 / \tau_{\text {in }} \equiv \mathbf{n}<$ $\sigma_{\text {in }}\left(v_{T}\right)_{n}>$. From equation (12), the ratio $n_{i} / n$ is crucial for estimating the typical drift time $\tau_{d}=R / v_{d}$. The original application was to super-critical $H I$ clouds, with dust obscuring the galactic $u-v$ ionizing radiation. It was thought that $n_{j} / n$ would fall rapidly to such low values that $\tau_{d}$ would become less than the free-fall time $\tau_{f}$, so that before even a super-critical cloud could begin its collapse, the bulk of the flux threading it would leak out to join the local galactic field, with the excess energy in the cloud field - supplied originally from the cloud's gravitational field and released as the field lines straighten - being thermalised through the ion-neutral friction. Subsequent estimates using a revised value for $\sigma_{\text {in }}$ (Osterbrock 1961) and taking account of ionization by the galactic cosmic ray flux showed that in typical HI clouds $\tau_{\mathrm{d}}$ is certainly much longer than $\tau_{f}$ and may exceed the Hubble time. In molecular clouds $n_{i} / n$ is significantly lower, but recent detailed discussions (Nakano \& Umebayashi 1986a,b; Norman \& Heyvaerts 1985; Nakano 1988) suggest that $\tau_{d}$ is still longer than $\tau_{f}$ by a factor 10 or more.

In constructing detailed models of cloud evolution under flux leakage, it should be remembered that the results may be sensitive to choice of geometry; in particular, the adoption on grounds of simplicity of plane-parallel symmetry is very dubious, as the gravitational field then saturates as the neutral particles settle, instead of increasing like $1 / \mathrm{r}^{2}$. Prima facie, there seems no reason to question Nakano's conclusion that up to densities of $10^{11} \mathrm{~cm}^{-3}$, flux leakage is fast enough to affect cloud evolution, but still much slower than free-fall, so that magnetic forces remain dynamically significant. It was early recognized (Mestel 1965) that moderately slow flux leakage can be important in super-critical, rapidly rotating clouds, if $\tau_{d}$ is competitive with the time of contraction due to magnetic transport of angular momentum: the leakage can assist fragmentation that may otherwise be inhibited by centrifugal forces, especially when the magnetic and rotation axes are nearly orthogonal, but the sub-condensations forming are still "strongly magnetic". More recently, Nakano $(1976,1983)$ and Mouschovias (1987) have emphasized that the sensitivity of flux-leakage to the value of $n_{i} / n$ can lead to a new fragmentational instability within a sub-critical cloud. In a dense cloud core the local drift time can be less than that from the cloud as a whole; the consequent adjustment of the core to magneto-gravitational equilibrium increases the density, leading to a further reduction in $\mathrm{n}_{\mathrm{i}} / \mathrm{n}$ and so to further differential flux leakage within the cloud. The picture is essentially the same as that given above but on a smaller scale, with the 
cloud core steadily losing flux to the rest of the cloud, instead of the cloud's losing flux to the intercloud medium. Thus an initially sub-critical core becomes super-critical, with its mass determined by ambipolar diffusion. The process is simply illustrated in some new studies on disc-like models with steadily increasing central mass concentration (Barker \& Mestel 1989). The transition of the core into gravitational collapse will be assisted by the associated decline in the level of the Alfvénic turbulence that contributes to cloud support in a realistic model (Lizano \& Shu 1989).

\section{Angular momentum}

A great advantage of flux leakage being slow even in a lightly ionized molecular cloud is that the strong field can transport away efficiently most of the embarrassingly high angular momentum that a condensation would inherit from both the galactic rotation and the interstellar turbulence (which would prevent a body of stellar mass from reaching anything like main-sequence densities). For brevity, we speak of rotation and contraction of a "cloud", but the transport can be from a cloud to the inter-cloud medium or from a cloud core to the outer parts of the cloud. The process is a simple application of basic magnetohydrodynamics. A contracting cloud initially in corotation with its surroundings will tend to spin up, so generating a shear along a line of a poloidal field $\underset{\sim}{B_{p}}$ linking the cloud with its surroundings; the consequent toroidal field $\underset{\sim}{B_{t}}$ propagates into and away from the cloud as a torsional Alfvén wave which tries to restore corotation. A rough estimate for the characteristic braking time $\tau_{b}$ is given by the time of travel of the waves through a surrounding region of density $\rho_{0}$ and with the same moment of inertia as the cloud. The results are to some extent model-dependent (Mouschovias \& Paleologou 1979, 1980; Mestel \& Paris 1984), but for a near-critical cloud with roughly radial field lines linking the cloud and the background field ${\underset{\sim}{\mathrm{B}}}_{0}$,

$\tau_{b} \simeq\left(M / \rho_{0}^{\frac{1}{2}} F\right)\left(R / R_{0}\right)^{6 / 5}, \quad R_{0}=\left(F / \pi B_{0}\right)^{\frac{1}{2}}$.

Compared with the instantaneous free-fall time $\tau_{f}$

$\tau_{b} / \tau_{f} \simeq\left(M / M_{c}(F)\right)\left(R_{0} / R\right)^{3 / 10 \simeq M / M}$.

Thus the ratio $M / M_{c}$ plays a double role: it not only discriminates between sub-critical and super-critical clouds, but it determines whether the braking time is less than or greater than the free-fall time. In case $A, M>M_{C}(F)$ initially, and a cloud with the expected initial angular momentum will contract in approximate magneto-centrifugo-gravitational balance, at the rate fixed by the braking time $\tau_{b}$ (as long as $\tau_{d}>>\tau_{b}$ ). In case $B, M<M_{c}(F)$ initially, and corotation with the surroundings is achieved rapidly, but the degree of contraction is limited to $R$ given roughly by equation (5). Under slow flux leakage $M_{c}$ decreases and the cloud begins by contracting quasi-statically in the time $\tau_{\mathrm{d}}>\tau_{\mathrm{f}}>\tau_{\mathrm{b}}$, with corotation being maintained and the ratio of centrifugal acceleration to gravity consequently declining. When $R / R_{0}$ has shrunk to $\simeq 2 \tau_{f} / \tau_{d}$, with $M \simeq M_{c}$, the subsequent contraction becomes dynamic (Nakano 1989). Once $M / M_{c}>1$, again (14) suggests that braking becomes less and less able to compete with spin-up consequent on the onset of free-fall, and the slowly rotating cloud will ultimately achieve magneto-centrifugo-gravitational balance, but at much higher densities. Again, further rotational evolution may occur at the rate determined by magnetic braking. 
Detailed analytical and numerical work (Gillis, Mestel \& Paris 1974, 1979; Mestel \& Paris 1979, 1984; Nakano 1989) confirms and refines these qualitative conclusions. There is also some tentative observational support, eg a correlation of higher rotation rates (40-50 times the usual values) in cloud cores with masses that are higher than the norm and presumed to be super-critical (see references in Shu et al. 1987).

The striking result is the sensitivity of the rotational history of the cloud to modest changes in the value of the parameter $f=F / \pi^{2} G \frac{1}{2} M$. In a cloud forming as a super-critical ( $f<f_{c}$ ) body, rotation may be dynamically important at all epochs, whereas in an initially sub-critical ( $f>f_{c}$ ) cloud, contracting though slow flux leakage, centrifugal forces may be kept low until the cloud or fragment reaches opaque densities. This contrasted behaviour is a further encouragement to pursue the idea of "bimodal star formation" (Shu et al. 1987; Lizano \& Shu 1989), suggested by the natural bifurcation into sub- and super-critical initial masses, paying particular attention to the joint effect of strong magnetic and centrifugal forces in the super-critical case.

The ability of the magnetic field to cope with the angular momentum problem prompts the question: is a magnetic field essential for star formation, so that the stars cannot form within a newly formed galaxy until the galactic dynamo has had time to operate? A definitive answer depends on further research into angular momentum transport in non-magnetic systems, eg via a spiral shock (Spruit 1987), or by shear instability ( $c f$. Miyama \& Sekiya 1988).

\section{The Masses of Proto-Stars}

A magnetic field that remains dynamically important all through the diffuse early stages of star formation can hardly fail to affect the expected mass spectrum. As already noted, in a cloud that is initially sub-critical, locally accelerated flux leakage from a dense core will weaken relative to self-gravitation both the Lorentz force exerted by the mean field and the associated Alfvenic turbulent support along the field, so that a super-critical core can develop, its mass depending on the details of the ambipolar diffusion process (Mouschovias 1987; Nakano 1988). An initially super-critical cloud - or indeed a super-critical core forming within a sub-critical cloud - can itself spontaneously fragment while retaining its flux if it is able to flatten along the field. Strong centrifugal forces (expected in an initially super-critical cloud) can inhibit such flattening when the magnetic and rotation axes are at a large angle, and fragmentation may again depend on flux leakage, with the resulting fragment masses tending to be high (Mestel 1965; Campbell \& Mestel 1987). When the axes are more nearly aligned, flattening will be limited by the strength of the Alfvénic turbulence. The minimum fragment mass that can condense in a uniformly dense cloud is estimated roughly as (15/2)(1 $\left.f^{2} / f_{c}\right)^{-1}\left(2 v_{t}{ }^{2} R / 3 G M\right) M$, where $M$ and $R$ are cloud mass and trans-field radius. If $v_{t}$ were just the thermal speed corresponding to $10-20 \mathrm{~K}$, this mass could be embarrassingly small, just as in the non-magnetic fragmentation problem (eg Low \& Lynden-Bell 1976). On the other hand, if $v_{t}{ }^{2}$ were maintained from external sources near the virial value $G M / R$, then both flattening and break-up would be strongly inhibited. The ultimate fragment mass spectrum should thus depend on the evolution of the Alfvenic turbulence, which in turn depends on the external energy supply, the cascading of energy from longer to shorter wavelengths, and dissipation through ion-electron collisions and shock formation. A decline in $v_{t}$ can lead to flattening and fragmentation without further flux leakage, especially if centrifugal 
forces limit overall contraction in two dimensions. Again, differential flux leakage from a cloud core, accompanied by spontaneous adjustment of $v_{t}$ to the changing local Alfvénic value $B_{c} /\left(4 \pi \rho_{c}\right)^{\frac{1}{2}}$ will allow formation of condensations of the order of the magnetic Jeans mass (1) with $B_{c}, \rho_{c}$ replacing $B_{0}, \rho_{0}$ (Lizano \& Shu 1989). Clearly much more work needs to be done, but one can plausibly hazard a guess that a strong magnetic flux retained through the whole of the diffuse phase will shift the mass spectrum towards higher values, a result that may be important for link-up between galactic dynamo and spiral arm theory (cf Mestel \& Subramanian, this volume and in preparation).

\section{Epoch of flux loss}

It appears that up to molecular cloud densities $n \sim 10^{11}$, direct flux leakage by

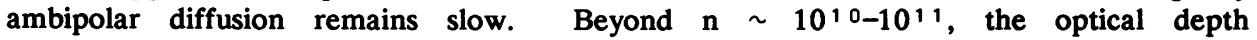
through a Jeans mass exceeds unity; during subsequent contraction the compressional heat generated is largely trapped, and the temperature rises nearly adiabatically. Beyond $T=10^{3} \mathrm{~K}$ thermal ionization increases $n_{i} / n$ to the level where flux freezing is again a very good approximation. In the interval $10<\mathrm{T}<10^{3}$, $\rho$ increases by $10^{4}$ or more. According to Nakano \& Umebayashi (1986b), in this interval the field no longer moves with the plasma. The electrons are largely attached to grains, and the current is carried by ions which suffer a large Ohmic dissipation due to ion-neutral collisions. The estimated remnant flux at $T=10^{3} \mathrm{~K}$ is less than that in the strongest magnetic Ap stars. However, the decline in the plasma density may sometimes be off-set from ionization by X-rays from nearby newly formed stars in the same cloud complex (Silk \& Norman 1983). A further possibility worthy of detailed study is that ionizing sub-cosmic rays may be generated locally as runaway particles during magnetic reconnection processes, as the field of a super-critical fragment detaches itself from the cloud field. And although we are concerned primarily with Population I stars, in the earliest epochs Population II and (possibly) Population III stars may have formed from magnetized gas at $T \simeq 10^{4} \mathrm{~K}$ (Hoyle 1953); collisional ionization will then have kept flux loss by large-scale ambipolar diffusion to a negligible rate at all epochs.

There are again advantages if the primeval flux remains dynamically important into the late phases of star formation within optically opaque clouds. Further magnetic redistribution of the remnant angular momentum may be significant in the formation of planetary and binary systems. Polarization observations of star-forming regions (Scarrott et al. and Warren-Smith et al. in Beck \& Gräve 1987) are plausibly an indicator of a magnetic field (though it is not clear whether the flux is a relic of the galactic field, or part of a dynamo-built field in a circumstellar disc). However, there is the observational constraint already noted, that all main sequence stars are magnetically weak, so that a proto-star entering the opaque phase with $f \sim f_{c}$ must certainly lose most of its flux subsequently. This may very well occur by magnetic buoyancy, in a thermal time-scale. As suggested by several authors, this reservoir of magnetic energy is a tempting source to power the pre-main sequence $T$ Tauri phenomenon.

\section{References}

Arons, J. \& Max, C. E., 1975. Astrophys. J., 196, L77.

Barker, D.M. \& Mestel, L., 1989. Mon. Not. R. astr. Soc. (submitted).

Beck, R. \& Gràve, R. (eds.) 1987. Interstellar Magnetic Fields, Springer, 


\section{Heidelberg}

Campbell, C.G. \& Mestel, L., 1987. Mon. Not. R. astr. Soc., 229, 549.

Chandrasekhar, S. \& Fermi, E., 1953. Astrophys. J., 118, 116.

Dewar, R.L., 1970. Phys. Fluids, 13, 2710.

Gillis, J., Mestel, L. \& Paris, R.B., 1974. Astrophys. Space Science, 27, 167.

Gillis, J., Mestel, L. \& Paris, R.B., 1979. Mon. Not. R. astr. Soc., 187, 311.

Hoyle, F., 1953. Astrophys. J., 118, 513.

Lizano, S. \& Shu, F.H., 1989. Astrophys. J., 342, 834.

Low, C. \& Lynden-Bell, D., 1976. Mon. Not. R. astr. Soc., 176, 367.

Mestel, L., 1965. Q. Jl. R. astr. Soc., 6, 161 and 265.

Mestel, L. \& Paris, R.B., 1979. Mon. Not. R. astr. Soc., 187, 337.

Mestel, L. \& Paris, R.B., 1984. Astron. Astrophys., 136, 98.

Mestel, L. \& Ray, T.P., 1985. Mon. Not. R. astr. Soc., 212, 275.

Mestel, L. \& Spitzer Jr., L., 1956. Mon. Not. R. astr. Soc., 116, 583.

Miyama, S.M. \& Sekiya, M., 1988. Prog. Theor. Phys. Supplement, 96, 95.

Mouschovias, T.C., 1974. Astrophys. J., 192, 37.

Mouschovias, T.C., 1976a, b. Astrophys. J., 206, 753; 207, 141.

Mouschovias, T.C., 1987. In Physical Processes in Interstellar Clouds, eds. Morfill, G.E. \& Scholer, M., Reidel, Dordrecht.

Mouschovias, T.C. \& Paleologou, E.V., 1979. Astrophys. J., 230, 204.

Mouschovias, T.C. \& Paleologou, E.V., 1980. Astrophys. J., 237, 877.

Myers, P.C. \& Goodman, A.A., 1988. Astrophys. J., 326, L27.

Nakano, T., 1976. Publs. astr. Soc. Japan, 28, 355.

Nakano, T., 1983. Publs. astr. Soc. Japan, 35, 209.

Nakano, T., 1988. In Galactic and Extragalactic Star Formation, eds. Pudritz, R.

\& Fich, M., Reidel, Dordrecht.

Nakano, T., 1989. Mon. Not. R. astr. Soc., in press.

Nakano, T. \& Umebayashi, T., 1986a, b. Mon. Not. R. astr. Soc., 218, 663; 221, 339.

Norman, C. \& Heyvaerts, J., 1985. Astron. Astrophys., 147, 247.

Osterbrock, D.E., 1961. Astrophys. J., 134, 270.

Parker, D.A., 1973. Mon. Not. R. astr. Soc., 163, 41.

Parker, D.A., 1974. Mon. Not. R. astr. Soc., 168, 331.

Parker, E.N., 1969. Space Sci. Rev., 9, 651.

Sargent, A.I., Beckwith, S., Keene, J. \& Masson, C., 1988. Astrophys. J., 333, 936.

Shu, F.H., Adams, F.C. \& Lizano, S., 1987. Ann. Rev. Astron. Astrophys., 25, 23.

Silk, J. \& Norman, C.A., 1983. Astrophys. J., 272, L49.

Spruit, H.C., 1987. Astron. Astrophys., 184, 173.

Strittmatter, P.A., 1966. Mon. Not. R. astr. Soc., 132, 359.

Sweet, P.A., 1950. Mon. Not. R. astr. Soc., 110, 69.

Tomisaka, K., Ikeuchi, S. \& Nakamura, T., 1988. Astrophys. J. 335, 239.

Zweibel, E.G. \& Josafatsson, K., 1983. Astrophys. J., 270, 511. 